\title{
A UNIFIED APPROACH TO UNIVALENCE CRITERIA IN THE UNIT DISC
}

\author{
MARTIN CHUAQUI
}

(Communicated by Albert Baernstein II)

\begin{abstract}
From the recent injectivity criterion of Osgood and Stowe we recover many of the known univalence criteria in the unit disc $D$ and derive as well new conditions on $D$ and simply-connected domains. While the criteria of Epstein can be established in this fashion, we show how the 'diameter term' in the criterion of Osgood and Stowe gives a sharper form of a condition of Ahlfors. Finally, on simply-connected domains we find a sufficient condition for univalence that is the counterpart to a necessary one proved by Bergman and Schiffer.
\end{abstract}

\section{INTRODUCTION}

The Schwarzian derivative has been a central concept in studying necessary and sufficient conditions for the univalence of analytic maps of a complex variable. In the unit disc, there is mainly one necessary condition for global univalence, proved originally by Kraus in 1932 and commonly attributed to Nehari, who discovered it independently in 1949 [Ne1]. His proof uses the so-called "area theorem", or equivalently, coefficient estimates for the power series expansion in the disc of the given analytic map. Another proof of this result was given by Bergman and Schiffer using the theory of kernel functions and conformal mappings [B-S]. Such an approach had the advantage that it could be applied to obtain similar results for other simply- and even multiply-connected domains. In the case of sufficient conditions for the univalence of analytic maps defined, say, in the unit disc, several apparently different criteria have been established. Since in many cases their proofs have relied on similar arguments, there has been an interest in deriving more general criteria of univalence. Also, frequently a stronger form of a given injectivity criterion can serve further as a sufficient condition for the existence of quasiconformal extensions to the entire plane. As a main step in understanding the phenomenon of injectivity and eventually quasiconformal extension, Epstein has proved recently a remarkable theorem which generalizes many such known results [Ep]. His approach is mainly differential geometric and uses in a beautiful way the geometry of hyperbolic 3-space. In quite a different character and, in a way, with a more

Received by the editors October 26, 1992 and, in revised form, May 7, 1993.

1991 Mathematics Subject Classification. Primary 30C45. 
classical approach, Anderson and Hinkkanen established also recently an even stronger sufficient criterion for univalence and quasiconformal extension [A-H]. Their theorem is more general, in that it applies to analytic maps defined on quasidiscs.

A generalization of the notion of Schwarzian derivative to higher dimensions was considered by Ahlfors in [Ah2]. He discusses that concept for local diffeomorphisms in $R^{n}, n \geq 3$, by making an analogy with the real and imaginary parts of the usual Schwarzian derivative of analytic maps in the plane. In euclidean space of dimension $\geq 3$, all conformal maps are Möbius transformations and, as in the case $n=2$, their Schwarzian derivative vanishes identically. So in some sense, the conformal significance of the Schwarzian derivative in $R^{n}$ is trivial for $n \geq 3$. In their paper The Schwarzian derivative and conformal mappings of Riemannian manifolds [O-S1], Osgood and Stowe introduce a notion of Schwarzian derivative on manifolds, which generalizes the classical operator in the plane. In their subsequent paper $A$ generalization of Nehari's univalence criterion [O-S2], and using their new notion, these authors establish a sufficient condition for the injectivity of a conformal local diffeomorphism of an $n$-dimensional Riemannian manifold $M$ to the standard sphere $S^{n}$. The idea of their proof, which can be partially traced back to some classical proofs, is to translate a given inequality on the Schwarzian to a differential inequality along geodesics. Then they apply a standard Sturm comparison theorem for ordinary differential equations. They obtain as corollaries, with $M$ the unit disc in the plane and particular choices of its metric, two well-known criteria of Nehari.

A slightly modified form of the Schwarzian derivative in [O-S1] appears in the recent work of $\mathrm{K}$. Carne. For this and other interesting ideas we refer the reader to $[\mathrm{Ca}]$.

The purpose of this paper is to derive from the general theorem of Osgood and Stowe some new and most of the known injectivity criteria. In particular, we shall obtain in this fashion the injectivity result of Epstein. The theorem in [O-S2] is stated as an inequality between the norm of the generalized Schwarzain and two geometric quantities of the base metric: its curvature and diameter. We show in this paper how a condition of Ahlfors can actually be improved when taking into account the diameter of the underlying metric. The language of conformal geometry in which the result of Osgood and Stowe is stated allows us to obtain a sufficient condition for the univalence of analytic maps defined on arbitrary simply-connected domains. This theorem can be considered as the counterpart to the necessary condition established by Bergman and Schiffer. Whether or not the sufficient condition exists on multiply-connected domains is still unknown.

\section{Preliminaries}

In this section we will set up notation and present enough of the work of Osgood and Stowe in [O-S1] so that we can state their injectivity theorem.

Let $M$ be an $n$-dimensional Riemannian manifold with metric $g$. When $M=R^{r}$, we will denote by $g_{0}$ the euclidean metric and $g_{1}$ will stand for the standard metric on the sphere $S^{n}$. Given a conformal metric $\hat{g}=e^{2 \varphi} g$ on $M$, Osgood and Stowe define the Schwarzian tensor of $\hat{g}$ with respect to $g$ as 
the symmetric, trace free $(0,2)$-tensor

$$
B_{g}(\varphi)=\operatorname{Hess}(\varphi)-d \varphi \otimes d \varphi-\frac{1}{n}\left(\Delta \varphi-|\operatorname{grad} \varphi|^{2}\right) g,
$$

where the metric dependent quantities on the right-hand side are computed with respect to the metric $g$. When $\psi$ is a conformal local diffeomorphism of $(M, g)$ to another Riemannian manifold $\left(N, g^{\prime}\right)$, then

$$
\psi^{*}\left(g^{\prime}\right)=e^{2 \varphi} g
$$

with $\varphi=\log |D \psi|$. The Schwarzian derivative of $\psi$ is defined by

$$
S_{g}(\psi)=B_{g}(\varphi) .
$$

For an analytic map $\psi$ in the plane with $g=g^{\prime}=g_{0}, \varphi=\log \left|\psi^{\prime}\right|$, and computing in standard coordinates one gets

$$
S_{g}(\psi)=\left(\begin{array}{rr}
\operatorname{Re}\{\psi, z\} & -\operatorname{Im}\{\psi, z\} \\
-\operatorname{Im}\{\psi, z\} & -\operatorname{Re}\{\psi, z\}
\end{array}\right)
$$

where $\{\psi, z\}=\left(\frac{\psi^{\prime \prime}}{\psi^{\prime}}\right)^{\prime}-\frac{1}{2}\left(\frac{\psi^{\prime \prime}}{\psi^{\prime}}\right)^{2}$ is the classical Schwarzian derivative.

On $M$, the conformal metric $\hat{g}=e^{2 \varphi} g$ is called Möbius with respect to $g$ if $B_{g}(\varphi)=0$, and so a conformal local diffeomorphism $\psi$ is said to be Möbius if $S_{g}(\psi)=0$. If $\varphi$ and $\sigma$ are smooth functions on $M$, then there is an important identity:

$$
B_{g}(\varphi+\sigma)=B_{g}(\varphi)+B_{\hat{g}}(\sigma),
$$

where $\hat{g}=e^{2 \varphi} g$. In a chain of conformal local diffeomorphisms $\psi_{1}:(M, g) \rightarrow$ $\left(N_{1}, g^{\prime}\right)$ and $\psi_{2}:\left(N_{1}, g^{\prime}\right) \rightarrow\left(N_{2}, g^{\prime \prime}\right)$, equation (1.2) can be formulated as

$$
S_{g}\left(\psi_{2} \circ \psi_{1}\right)=S_{g}\left(\psi_{1}\right)+\psi_{1}^{*}\left(S_{g^{\prime}}\left(\psi_{2}\right)\right) \text {. }
$$

This reduces to the classical formula for the Schwarzian derivative of a composition of analytic maps in the plane.

By $\left\|B_{g}(\varphi)\right\|$ we mean the norm of the Schwarzian tensor $B_{g}(\varphi)$ with respect to $g$, as a bilinear form on each tangent space, that is,

$$
\left\|B_{g}(\varphi)\right\|=\max \left\{\left|B_{g}(\varphi)(X, Y)\right|:|X|=|Y|=1\right\} .
$$

With this, we now present the result in [O-S2].

Theorem 1.1. Let $(M, g)$ be a Riemannian manifold of dimension $n \geq 2$ and $\psi:(M, g) \rightarrow\left(S^{n}, g_{1}\right)$ a conformal local diffeomorphism. Suppose that the scalar curvature of $M$ is bounded above by $n(n-1) K$ for some $K \in R$, and that any two points in $M$ can be joined by a geodesic of length $<\delta$ for some $0<\delta \leq \infty$. If

$$
\left\|S_{g}(\psi)\right\| \leq \frac{2 \pi^{2}}{\delta^{2}}-\frac{1}{2} K
$$

then $\psi$ is injective.

With $M$ the unit disc in the plane and $g$ alternately the euclidean and hyperbolic metric, Osgood and Stowe derive from this theorem the classical 
criteria of Nehari, namely, that

$$
|\{\psi, z\}| \leq \frac{\pi^{2}}{2} \quad \text { or } \quad|\{\psi, z\}| \leq \frac{2}{\left(1-|z|^{2}\right)^{2}}, \quad \text { all } z \in D
$$

implies that $\psi$ is univalent.

We point out that Theorem 1.1 can be stated with $\left(S^{n}, g_{1}\right)$ replaced by $\left(R^{n}, g_{0}\right)$ or $H^{n}$ with its metric of constant negative curvature. This follows from the transformation law (1.2) and the fact that both $g_{1}$ and the hyperbolic metric are Möbius with respect to the euclidean metric. Finally, let $\operatorname{scal}(g)$ be the scalar curvature of $g$. It is easy to verify that the proof given by Osgood and Stowe works equally well only assuming that at each point in $M$ the norm of the Schwarzian derivative of $\psi$ is bounded above by

$$
\frac{2 \pi^{2}}{\delta^{2}}-\frac{\operatorname{scal}(g)}{2 n(n-1)} \text {. }
$$

This is the form of the theorem we shall use to derive the various injectivity criteria in the unit disc.

\section{INJECTIVITY CRITERIA IN THE UNIT DISC}

Throughout this section, $D$ will denote the open unit disc in the plane. We will apply Theorem 1.1 on $D$ with metrics conformal to the euclidean metric $g_{0}$ which are of the form

$$
g=\frac{e^{2 \sigma}}{\left(1-|z|^{2}\right)^{2 t}} g_{0}, \quad t>0
$$

Epstein's general theorem of univalence in [Ep] will follow by setting $t=1$, and an important case will be when $\sigma$ is harmonic. In order to apply Theorem 1.1 , we will require $g$ to have nonpositive curvature and also impose a growth condition on the coefficient $e^{\sigma}\left(1-|z|^{2}\right)^{-t}$ that will ensure that any two points in $D$ can be joined by a geodesic in the metric $g$.

Lemma 2.1. Let $g$ be as in (2.1). If for some $0<r<1$

$$
\left|\sigma_{z}(z)\right| \leq \frac{t|z|}{1-|z|^{2}}
$$

for all $r \leq|z|<1$, then any two points in $D$ can be joined by a geodesic in the metric $g$.

Proof. Let $\eta=\sigma-t \log \left(1-|z|^{2}\right)$. Then (2.2) guarantees that the radial derivative of $\eta$ is nonegative for $|z| \geq r$. Given now two points $x, y \in D$, we seek a geodesic in the metric $g$ joining $x$ and $y$. Let $d=\inf L_{g}(\gamma)$, where the infimum is taken over all smooth curves $\gamma$ in $D$ that join $x$ to $y$, and $L_{g}(\gamma)$ is the length of $\gamma$ in $g$. Under the hypothesis of the lemma, the conformal factor $e^{\eta}$ eventually increases as one approaches $\partial D$. Hence, given $\epsilon>0$, there exists a compact set $K \subset D$ such that any curve $\gamma$ joining $x$ to $y$ with $L_{g}(\gamma)<d+\epsilon$ is completely contained in $K$. We can therefore find a minimizing sequence $\left\{\gamma_{n}\right\}$ converging to the desired geodesic.

We now state the main result in this section. 
Theorem 2.2. Let $g$ as in (2.1) have negative curvature and satisfy (2.2). Let $\delta \leq \infty$ be the diameter of $(M, g)$, and let $\psi$ be analytic and locally injective in $D$. If

$$
\left|\frac{\left(1-|z|^{2}\right)^{2}\left(\sigma_{z z}-\sigma_{z}^{2}-\frac{1}{2}\{\psi, z\}\right)-2 t \bar{z}\left(1-|z|^{2}\right) \sigma_{z}+t(1-t) \bar{z}^{2}}{t+\left(1-|z|^{2}\right)^{2} \sigma_{z z}}\right|
$$

$$
\leq 1+\frac{\pi^{2} e^{2 \sigma}\left(1-|z|^{2}\right)^{2(1-t)}}{\delta^{2}\left(t+\left(1-|z|^{2}\right)^{2} \sigma_{z z}\right)}
$$

then $\psi$ is univalent.

In general, $\delta=\infty$ for $t \geq 1$. On the other hand, for $t<1$ and certain choices of $\sigma$, the diameter $\delta$ will be finite. We will come back to this point later.

Proof. As mentioned before, we shall derive (2.3) from Theorem 1.1 by computing the Schwarzian derivative of $\psi$ in the metric $g$. The scalar curvature of $g$ is given by

$$
k=-8 e^{-2 \eta} \eta_{z z}=-8 e^{2 \eta}\left(t+\left(1-|z|^{2}\right)^{2} \sigma_{z z}\right),
$$

where, as before, $\eta=\sigma-t \log \left(1-|z|^{2}\right)$. We also remind the reader that in this case the scalar curvature equals twice the standard Gaussian curvature.

Let $\varphi=\log \left|\psi^{\prime}\right|$. Then

$$
\psi^{*}\left(g_{0}\right)=e^{2 \varphi} g_{0}=e^{2(\varphi-\eta)} g,
$$

and we therefore have to compute $B_{g}(\varphi-\eta)$. Using the addition formula (1.2), we have

$$
B_{g}(\varphi-\eta)=B_{g}(-\eta)+B_{g_{0}}(\varphi)
$$

and since

$$
0=B_{g}(\eta-\eta)=B_{g}(-\eta)+B_{g_{0}}(\eta)
$$

we conclude that

$$
B_{g}(\varphi-\eta)=B_{g_{0}}(\varphi)-B_{g_{0}}(\eta)
$$

By definition

$$
\left\|B_{g}(\varphi-\eta)\right\|=e^{-2 \eta}\left\|B_{g}(\varphi-\eta)\right\|_{g_{0}}=e^{-2 \eta}\left\|B_{g_{0}}(\varphi)-B_{g_{0}}(\eta)\right\|_{g_{0}} .
$$

Computing in standard coordinates, $B_{g_{0}}(\varphi)-B_{g_{0}}(\eta)$ is a matrix of the form

$$
\left(\begin{array}{rr}
\alpha & \beta \\
\beta & -\alpha
\end{array}\right)
$$

and its euclidean norm is $|\alpha+i \beta|$. By $(1.1), B_{g_{0}}(\varphi)$ will be represented by $\overline{\{\psi, z\}}$ and $B_{g_{0}}(\eta)$ will be given by $A+i B$, where

$$
\begin{aligned}
& A=\eta_{x x}-\eta_{x}^{2}-\frac{1}{2}\left(\eta_{x x}+\eta_{y y}-\eta_{x}^{2}-\eta_{y}^{2}\right), \\
& B=\eta_{x y}-\eta_{x} \eta_{y} .
\end{aligned}
$$


A straightforward calculation yields

$$
\overline{A+i B}=2 \sigma_{z z}-2 \sigma_{z}^{2}-\frac{4 t \bar{z} \sigma_{z}}{1-|z|^{2}}+\frac{2 t(1-t) \bar{z}^{2}}{\left(1-|z|^{2}\right)^{2}} .
$$

Theorem 1.1 as in the remark after its statement in the last section reads as

$$
\left\|S_{g}(\psi)\right\| \leq \frac{2 \pi^{2}}{\delta^{2}}+2 \frac{t+\left(1-|z|^{2}\right) \sigma_{z \bar{z}}}{e^{2 \eta}}
$$

From this and the last computations, we obtain (2.3).

Setting $t=1$ in (2.3) we obtain:

Corollary 2.3 (Epstein). Let $g$ as in (2.1) have negative curvature and satisfy (2.2). If $\psi$ is analytic and locally injective in $D$ and satisfies

$$
\left|\frac{\left(1-|z|^{2}\right)^{2}\left(\sigma_{z z}-\sigma_{z}^{2}-\frac{1}{2}\{\psi, z\}\right)-2 \bar{z}\left(1-|z|^{2}\right) \sigma_{z}}{1+\left(1-|z|^{2}\right)^{2} \sigma_{z \bar{z}}}\right| \leq 1,
$$

then $\psi$ is univalent.

Notice that we have left out the second term in the right-hand side of (2.3). As we shall see next, there are important cases when including the diameter term constitutes an actual improvement of known criteria.

Let $\sigma=0$ in (2.3), which clearly satisfies (2.1). Then $\delta=\infty$ if $t \geq 1$, but for $t<1$

$$
\delta=2 \int_{0}^{1} \frac{d x}{\left(1-x^{2}\right)^{t}}
$$

This integral can be expressed in terms of the $\Gamma$-function as

$$
\delta=\pi^{\frac{1}{2}} \frac{\Gamma(1-t)}{\Gamma\left(\frac{3}{2}-t\right)}
$$

Corollary 2.4. Let $\psi$ be analytic and locally univalent in $D$. If either

$$
\left|\{\psi, z\}-\frac{2 t(1-t)}{\left(1-|z|^{2}\right)^{2}} \bar{z}^{2}\right| \leq \frac{2 t}{\left(1-|z|^{2}\right)^{2}}, \quad t \geq 1,
$$

or

$$
\left|\{\psi, z\}-\frac{2 t(1-t)}{\left(1-|z|^{2}\right)^{2}} \bar{z}^{2}\right| \leq \frac{2 t}{\left(1-|z|^{2}\right)^{2}}+\frac{2 \pi}{\left(1-|z|^{2}\right)^{2 t}}\left(\frac{\Gamma\left(\frac{3}{2}-t\right)}{\Gamma(1-t)}\right)^{2}, \quad t<1,
$$

then $\psi$ is injective.

If we let $t=2$ in (2.4), then we obtain

$$
\left|\{\psi, z\}+\frac{4 \bar{z}^{2}}{\left(1-|z|^{2}\right)^{2}}\right| \leq \frac{4}{\left(1-|z|^{2}\right)^{2}}
$$

as a sufficient condition for $\psi$ to be univalent. This can be used to give another proof of the criterion announced by Pokornyi and proved by Nehari [Ne2], 
namely, that

$$
|\{\psi, z\}| \leq \frac{4}{1-|z|^{2}}
$$

implies the univalence of $\psi$. Indeed, (2.7) implies (2.6) as

$$
\left|\{\psi, z\}+\frac{4 \bar{z}^{2}}{\left(1-|z|^{2}\right)^{2}}\right| \leq \frac{4}{1-|z|^{2}}+\frac{4|z|^{2}}{\left(1-|z|^{2}\right)^{2}}=\frac{4}{\left(1-|z|^{2}\right)^{2}} .
$$

The criteria (2.4) for $1 \leq t \leq 2$ and (2.5) without the diameter term correspond to Ahlfors's condition

$$
\left|\{\psi, z\}-\frac{2 c(1-c) \bar{z}^{2}}{\left(1-|z|^{2}\right)^{2}}\right| \leq \frac{2|c|}{\left(1-|z|^{2}\right)^{2}}
$$

when $c$ is real. In (2.8) $c$ may be any complex number with $|c-1|<1$ [Ah1]. We mention here the interesting paper of Anderson and Hinkkanen [A-H], who derive from their general theorem on univalence, among several others, both criteria of Ahlfros in [Ah1]. We would like to show that there exist (univalent) functions that satisfy (2.5) as stated and which would not do so without the diameter term. Notice first that inequality (2.5) interpolates the conditions of Nehari:

$$
|\{\psi, z\}| \leq \frac{\pi^{2}}{2} \quad \text { and } \quad|\{\psi, z\}| \leq \frac{2}{\left(1-|z|^{2}\right)^{2}},
$$

which are obtained from (2.5) as limiting cases when $t \rightarrow 0$ and $t \rightarrow 1$.

Both of Nehari's criteria are sharp, with extremal functions that are geometrically simple: they are given respectively by

$$
f(z)=e^{\pi z} \text { and } f(z)=\log \frac{1+z}{1-z} .
$$

Thus the following problem seems of interest to us: determine whether or not the interpolating criteria are sharp as well, and if they are, try to choose simple extremal functions varying smoothly in $t$. So far we have been able to resolve the case when $t \geq \frac{1}{2}$. The construction will also yield an extremal function for (2.4) when $1 \leq t \leq 2$. For $0<t<1$ let

$$
\psi_{t}(z)=\exp \left\{\frac{2 \pi i}{\delta} \phi_{t}(z)\right\}
$$

where

$$
\phi_{t}(z)=\int_{0}^{z} \frac{d \zeta}{\left(1-\zeta^{2}\right)^{t}}
$$

and

$$
\delta=2 \int_{0}^{1} \frac{d x}{\left(1-x^{2}\right)^{t}}
$$

It is easy to verify that $\phi_{t}$ maps the unit disc onto a bounded, convex, symmetric domain centered at the origin. The function $\psi_{t}$ is univalent in the open unit 
disc and $\psi_{t}(1)=\psi_{t}(-1)=-1$. A short calculation gives

$$
\left\{\psi_{t}, z\right\}=\frac{2 t(1-t)}{\left(1-z^{2}\right)^{2}} z^{2}+\frac{2 t}{\left(1-z^{2}\right)^{2}}+\frac{2 \pi}{\left(1-z^{2}\right)^{2 t}}\left(\frac{\Gamma\left(\frac{3}{2}-t\right)}{\Gamma(1-t)}\right)^{2} .
$$

The function $\phi_{t}$ has Schwarzian derivative equal to

$$
\left\{\phi_{t}, z\right\}=\frac{2 t(1-t)}{\left(1-z^{2}\right)^{2}} z^{2}+\frac{2 t}{\left(1-z^{2}\right)^{2}}
$$

and is well defined for all values of $t$. For $t>1, \phi_{t}$ maps 1 and -1 to the point at infinity. We claim that $\psi_{t}$ satisfies (2.5) (only) when $\frac{1}{2} \leq t<1$ and that $\phi_{t}$ satisfies (2.4) when $1 \leq t \leq 2$.

\section{Lemma 2.5. The inequality}

$$
\left|\frac{2 t(1-t)}{\left(1-z^{2}\right)^{2}} z^{2}+\frac{2 t}{\left(1-z^{2}\right)^{2}}-\frac{2 t(1-t)}{\left(1-|z|^{2}\right)^{2}} \bar{z}^{2}\right| \leq \frac{2 t}{\left(1-|z|^{2}\right)^{2}}
$$

holds for all $|z|<1$ if and only if $\frac{1}{2} \leq t \leq 2$.

Proof. Let $z=x+i y$. It is clear that (2.9) holds when $z=x$ is real, and by symmetry it suffices to check it when $y>0$. We will analyze the inequality along the arcs of circles

$$
x^{2}+(y+\lambda)^{2}=1+\lambda^{2}, \quad \lambda>0,
$$

that lie in the unit disc. These circles are centered at $(0,-\lambda)$ and pass through the points $( \pm 1,0)$. Any point $z \in D$ with $y>0$ is contained in one of these arcs.

In (2.9) divide by $2 t$ and multiply by $\left(1-|z|^{2}\right)^{2}\left|1-z^{2}\right|^{2}$. This leads to the inequality

$$
\left|(1-t)\left\{z^{2}\left(1-|z|^{2}\right)^{2}-\bar{z}^{2}\left(1-z^{2}\right)^{2}\right\}+\left(1-|z|^{2}\right)^{2}\right| \leq\left|1-z^{2}\right|^{2} .
$$

The expression $z^{2}\left(1-|z|^{2}\right)^{2}-\bar{z}^{2}\left(1-z^{2}\right)^{2}$, which can be rewritten as the expression $2|z|^{2}\left(|z|^{2}-z^{2}\right)+z^{2}-\bar{z}^{2}$, has real part $2|z|^{2}\left(|z|^{2}-x^{2}+y^{2}\right)=4|z|^{2} y^{2}$ and imaginary part $4 x y\left(1-|z|^{2}\right)$. Using (2.10) we can write

$$
\left|1-z^{2}\right|^{2}=4 y^{2}\left(1+\lambda^{2}\right) \text { and }\left(1-|z|^{2}\right)^{2}=4 \lambda^{2} y^{2} \text {. }
$$

We insert this in (2.11) and divide by $4 y^{2}$ to obtain

$$
\left.|(1-t)| z\right|^{2}+\lambda^{2}+2 i(1-t) \lambda x \mid \leq 1+\lambda^{2} \text {. }
$$

Square both sides and let $s=1-t$. After cancelling $\lambda^{4}$ this yields

$$
\left(4 s^{2} x^{2}+2 s|z|^{2}\right) \lambda^{2}+s^{2}|z|^{4} \leq 1+\lambda^{2} \text {. }
$$

Since $\lambda>0$ can be arbitrarily large and since the left-hand side of inequality (2.12) is maximized by letting $x \rightarrow 1$ (along the arc), we conclude that in order for the original inequality (2.9) to hold we need $4 s^{2}+2 s \leq 2$. This will be the case precisely when $-1 \leq s \leq \frac{1}{2}$, i.e., $\frac{1}{2} \leq t \leq 2$. For such values of $t$ the constant term $s^{2}|z|^{4}$ in (2.12) will be less than 1. This finishes the proof of the lemma. 
It follows from the triangle inequality that $\psi_{t}$ will satisfy $(2.5)$ for $\frac{1}{2} \leq t<1$. The proof of the failure of (2.9) when $t<\frac{1}{2}$ and the fact that the diameter term is of lower order than $\left(1-|z|^{2}\right)^{-2}$ shows that $\psi_{t}$ will not satisfy (2.5) for these values of $t$. Finally, for any $0<t<1 \quad \psi_{t}$ can never satisfy (2.5) without the diameter term: simply take $z=x$ real.

For $\frac{1}{2} \leq t<1$ the criteria (2.5) are also sharp in the following sense: given any constant $c>1$ there exists an $\epsilon>0$ small enough so that the non-univalent function

$$
\exp \left\{(1+\epsilon) \frac{2 \pi i}{\delta} \phi_{t}(z)\right\}
$$

satisfies (2.5) with the right-hand side multiplied by $c$. This can be seen from the proof of Lemma 2.5.

If $1 \leq t \leq 2$, then the functions $\phi_{t}$ are extremals for (2.4). The failure of this for $t>2$ can at least be explained now; unlike the maps $\psi_{t}$, which remain univalent for all $0<t<1, \phi_{t}$ is not injective when $t>2$ : the order of blow up at \pm 1 is $t-1>1$, hence the image of small (almost) half circles centered at \pm 1 have to overlap at infinity. A better understanding of both criteria in Corollary 2.4 in the ranges $0<t<\frac{1}{2}$ and $t>2$ has eluded us.

It is unlikely that in the complete generality of Theorem 2.2 one will be able to determine whether the criteria with the diameter term are indeed better than the ones without it. Any theorem along these lines characterizing classes of functions $\sigma$ (and ranges for $t$ ) would be in our opinion very interesting. Perhaps as a motivation for this we bring up a second condition of Ahlfors:

$$
\left.\left|z \frac{\psi^{\prime \prime}}{\psi^{\prime}}\left(1-|z|^{2}\right)+c\right| z\right|^{2} \mid \leq 1
$$

with $|c|<1, c$ complex [Ah2].

For real values of $c(2.13)$ can be deduced from Theorem 2.2 by letting $\sigma=\log \left|\psi^{\prime}\right|$. Then $\sigma$ is harmonic and $\sigma_{z z}-\sigma_{z}^{2}=\frac{1}{2}\{\psi, z\}$. In order to apply the theorem we need the condition in Lemma 2.1 to be satisfied, which in this case is

$$
\left|\frac{\psi^{\prime \prime}}{\psi^{\prime}}\right| \leq \frac{2 t}{1-|z|^{2}}
$$

for all $r \leq|z|<1$, for some $0<r<1$. Assuming this, Theorem 2.2 without the diameter term gives

$$
\left.\left|z \frac{\psi^{\prime \prime}}{\psi^{\prime}}\left(1-|z|^{2}\right)-(1-t)\right| z\right|^{2} \mid \leq 1
$$

as a sufficient condition for the univalence of $\psi$. In (2.15) $t$ may be any real number. Again, one would like to show that in general the diameter term cannot be left out. Notice that the omitted term now depends on $\left|\psi^{\prime}\right|$ (see Theorem 2.2) and that the diameter is likely to be infinite if $t \geq 1$. So far we have been unsuccessful here, but our attempts have led us to several extremal functions for (2.15). (By extremal we mean that they satisfy (2.15) but do not for any 
constant less than 1 on the right-hand side.) These functions are

$$
g_{1}(z)=\int_{0}^{z} \frac{d \zeta}{\left(1-\zeta^{2}\right)^{1-\frac{l}{2}}}, \quad g_{2}(z)=\int_{0}^{z}\left(1-\zeta^{2}\right)^{\frac{1}{2}} d \zeta
$$

and

$$
g_{3}(z)=\int_{0}^{z} \frac{\left(1+\zeta^{2}\right)^{\frac{1}{2}}}{\left(1-\zeta^{2}\right)^{\frac{1}{2}}} d \zeta, \quad g_{4}(z)=\int_{0}^{z} \frac{d \zeta}{\left(1+\zeta^{2}\right)^{\frac{l}{2}}} .
$$

The first two functions are extremals along the $x$-axis while the last two are along the $y$-axis. The idea has been to consider 'variations' $\psi=f(g)$ of the extremals to show that, for siutable $f, \psi$ will satisfy (2.15) only in the presence of the diameter term.

\section{THE SIMPLY CONNECTED CASE}

Here, we shall derive from Theorem 1.1 a sufficient condition for the univalence of a locally schlicht analytic map defined on a simply-connected domain $D_{1}$. This condition will come as a counterpart to the necessary condition for such global univalence established in [B-S], namely,

$$
\left|\frac{1}{\pi} U_{\psi}(z, z)+l(z, z)\right| \leq K(z, \bar{z}) .
$$

The terms involved are defined as follows: let

$$
U_{\psi}(z, \zeta)=\frac{\partial^{2}}{\partial \zeta \partial z} \log \frac{\psi(z)-\psi(\zeta)}{z-\zeta},
$$

so that

$$
U_{\psi}(z, z)=-\frac{1}{6}\{\psi, z\} .
$$

Let $h(z, \zeta)$ be the Green's function for the Dirichlet problem in $D_{1}$. Then

$$
K(z, \bar{\zeta})=-\frac{2}{\pi} \frac{\partial^{2}}{\partial z \partial \bar{\zeta}} h(z, \zeta)
$$

is the Bergman kernel, and the function $l$ is defined by

$$
l(z, \zeta)=\frac{1}{\pi} \frac{1}{(z-\zeta)^{2}}+\frac{2}{\pi} \frac{\partial^{2}}{\partial z \partial \zeta} h(z, \zeta)
$$

It is not dificult to see that the singularity of $h$ disappears when this function is differentiated as in the equation relating it to the kernel $K$. Also, by a theorem in [B-S], $l$ is actually regular in $D_{1}$.

Let $h_{0}, K_{0}$, and $l_{0}$ denote the corresponding quantities when $D_{1}=D$ is the unit disc. Since

one finds that

$$
h_{0}(z, \zeta)=\log \left|\frac{1-z \bar{\zeta}}{z-\zeta}\right|
$$

$$
K_{0}(z, \bar{\zeta})=\frac{1}{\pi}(1-z \bar{\zeta})^{-2}
$$

and

$$
l_{0}(z, \zeta)=0
$$


Thus on $D,(3.1)$ gives the criterion of Nehari, which states that

$$
|\{\psi, z\}| \leq \frac{6}{\left(1-|z|^{2}\right)^{2}}
$$

is necessary for the univalence of $\psi$.

Let now $F$ be a conformal diffeomorphism of $D_{1}$ onto $D$. Then,

$$
h(z, \zeta)=h_{0}(F(z), F(\zeta)),
$$

and in differentiating this equation one obtains

$$
K(z, \bar{\zeta})=K_{0}(F(z), \overline{F(\zeta)}) F^{\prime}(z) \overline{F^{\prime}(\zeta)}
$$

and

$$
l(z, \zeta)=-\frac{1}{\pi}\left(\frac{F^{\prime}(z) F^{\prime}(\zeta)}{(F(z)-F(\zeta))^{2}}-\frac{1}{(z-\zeta)^{2}}\right) .
$$

Hence, $(\pi K(z, \bar{z}))^{1 / 2}|d z|$ is the Poincare metric on $D_{1}$ and $l(z, z)=$ $\frac{1}{6 \pi}\{F, z\}$.

Theorem 3.1. The map $\psi$ is univalent if

$$
\left|\frac{1}{\pi} U_{\psi}(z, z)+l(z, z)\right| \leq \frac{1}{3} K(z, \bar{z}) .
$$

Proof. If $D=D_{1}$ is the unit disc, then (3.2) reduces to the classical condition of Nehari. For generic $D_{1}$ simply-connected, (3.2) is a reformulation of Nehari's criterion using the Schwarzian derivative of a composition and the conformal invariance of the kernel $K$.

The inequality (3.2) can also be derived from Theorem 1.1 with $M=D_{1}$ and $g=\pi K(z, \bar{z}) g_{0}$, its Poincaré metric. For this it suffices to show that

$$
\left\|S_{g}(\psi)\right\| \leq 2
$$

is equivalent to

$$
|\{\psi, z\}-\{F, z\}| \leq 2 \pi K(z, \bar{z}) .
$$

We observe first that the Poincare metric on $D$ is Möbius with respect to the euclidean metric. The addition formula for the Schwarzian tensor together with the conformal invariance of the Poincaré metric now give

$$
S_{g_{0}}(F)=B_{g_{0}}\left(\frac{1}{2} \log K\right) \text {. }
$$

Therefore,

$$
S_{g}(\psi)=S_{g_{0}}(\psi)-B_{g_{0}}\left(\frac{1}{2} \log K\right)=S_{g_{0}}(\psi)-S_{g_{0}}(F) .
$$

In light of the considerations that will follow, we prefer to think of (3.2) as a restatement of Theorem 1.1 on the domain $D_{1}$ with a particular choice of conformal metric. As mentioned in the introduction, (3.1) holds also as a necessary condition for univalence on multiply-connected domains. It was natural to seek a corresponding sufficient condition on such domains by using Theorem 1.1 probably with the Bergman metric $(\pi K(z, \bar{z}))^{1 / 2}|d z|$. However, in [Ch3] we prove : 
Theorem 3.2. Let $(M, g)$ be a complete Riemannian manifold of dimension $n \geq 2$ and $\psi:(M, g) \rightarrow\left(S^{n}, g_{1}\right)$ a conformal local diffeomorphism. If

$$
\left\|S_{g}(\psi)\right\| \leq-\frac{\operatorname{scal}(g)}{2 n(n-1)}
$$

then $M$ is simply-connected.

Since the Bergman metric is complete, a criterion of injectivity derived from Theorem 1.1 in this metric would have to hold with $\delta=\infty$. The curvature term is controlled since the Gaussian curvature of the Bergman metric is $\leq-4$ [Sa]. Whether or not the inequality (3.2) corresponds to Theorem 1.1 on a multiplyconnected domain with its Bergman metric we do not know. The difficulty lies in proving that the Schwarzian tensor of the Bergman metric with respect to $g_{0}$ is represented by the quantity $6 \pi l(z, z)$. This is equivalent to establishing the identity

$$
2\left(\varphi_{z z}-\varphi_{z}^{2}\right)=6 \pi l(z, z)
$$

where $\varphi=\frac{1}{2} \log K(z, \bar{z})$. If this were true, then by Theorem 3.2 no analytic function $\psi$ would ever satisfy (3.2) on a multiply-connected domain.

\section{ACKNOWLEDGMENTS}

The results of this paper formed part of the author's doctoral dissertation, completed at Stanford in 1990 under the supervision of Brad Osgood. The author would like to express his gratitude to him for his continued support and encouragement during those years.

The author also expresses his thanks for the referee's thoughtful suggestions concerning the exposition of the more relevant results presented here.

\section{REFERENCES}

[Ah1] L. V. Ahlfors, Sufficient conditions for quasiconformal extensions, Discontinuous Groups and Riemann Surfaces (Leon Greenberg, ed.), Ann. of Math. Stud., no. 79, Princeton Univ. Press, Princeton, NJ, 1974.

[Ah2] - Schwarzian derivative and cross-ratio in $R^{n}$, Complex Analysis: A Collection of Papers Dedicated to Albert Pluger, Birkhäuser, Boston, MA, 1989.

[A-H] J. M. Anderson and A. Hinkkanen, Univalence criteria and quasiconformal extensions, Trans. Amer. Math. Soc. 324 (1991), 823-842.

[B-S] S. Bergman and M. Schiffer, Kernel functions and conformal mappings, Compositio Math. 8 (1951), 205-249.

[Ca] K. Carne, Schwarzian derivative for conformal maps, J. Reine Angew. Math. 408 (1990), 10-33.

[Ch1] M. Chuaqui, The Schwarzian derivative and quasiconformal reflections on $S^{n}$, Ann. Acad. Sci. Fenn. Ser. A I Math. 17 (1992), 315-326.

[Ch2] - On a theorem of Nehari and quasidiscs, Ann. Acad. Sci. Fenn. Ser. A I Math. 18 (1993), 117-124.

[Ch3] - Ricci curvature and a criterion for simple-connectivity on the sphere, Proc. Amer. Math. Soc. (to appear).

[Ch-O1] M. Chuaqui and B. Osgood, The Schwarzian derivative and conformally natural quasiconformal extensions from one to two to three dimensions, Math. Ann. 292 (1992), 267-280.

[Ch-O2] _ Sharp distortion theorems associated with the Schwarzian derivative, J. London Math. Soc. (2) 48 (1993), 289-298. 
[Ep] C. Epstein, The hyperbolic Gauss map and quasiconformal reflection, J. Reine Angew. Math. 372 (1986), 96-135.

[Ne1] Z. Nehari, The Schwarzian derivative and schlicht functions, Bull. Amer. Math. Soc. 55 (1949), 545-551.

[Ne2] Univalence criteria depending on the Schwarzian derivative Illinois J. of Math. 23 (1979), 345-351.

[O-S1] B. Osgood and D. Stowe, The Schwarzian derivative and conformal mappings of Riemannian manifolds, Duke Math. J. 67 (1992), 57-99.

[O-S2] , A generalization of Nehari's univalence criterion, Comment. Math. Helv. 65 (1990), 234-242.

[Sa] M. Sakai The sub-mean value property of subharmonic functions and its applications to estimate the Gaussian curvature of the span metric, preprint.

Department of Mathematics, University of Pennsylvania, Philadelphia, PennsylvaNIA 19104

Current address: P. Universidad Católica de Chile, Casilla 306, Santiago 22, Chile

E-mail address: mchuaqui@mat.puc.cl 